\title{
Imaging of Nonischemic Cardiomyopathy
}

\author{
David A. Bluemke and Shawn D. Teague
}

\section{Learning Objectives}

- Identify nonischemic pattern of delayed enhancement.

- Develop a differential diagnosis for dilated cardiomyopathy.

- Evaluate the functional impact of nonischemic cardiomyopathy.

\subsection{Introduction}

Symptomatic myocardial disease not the result of flowlimiting coronary artery disease or prior myocardial infarction is commonly known as nonischemic cardiomyopathy. Clinical manifestations include arrhythmia, palpitations, heart failure, shortness of breath, lower extremity edema, syncope, and chest pain. Nonischemic cardiomyopathy is more common in women and younger patients. In general, the prognosis for nonischemic cardiomyopathy is better than for ischemic disease. There may be either systolic, diastolic, or combined heart failure. Infiltrative cardiomyopathies in particular tend to result in diastolic dysfunction.

In most patients, ischemic cardiomyopathy should be considered and excluded prior to further work-up of nonischemic cardiomyopathy. Echocardiography is the primary modality for evaluating left ventricular function as well as valvular and diastolic function. Advanced techniques including pulsed tissue Doppler and 3-D imaging have added diag-

\section{A. Bluemke}

Department of Radiology, University of Wisconsin School of Medicine and Public Health, Madison, WI, USA

e-mail: dbluemke@wisc.edu

S. D. Teague $(\bowtie)$

Department of Radiology, National Jewish Health, University

of Colorado School of Medicine, Denver, CO, USA

e-mail: teagues@njhealth.org nostic and prognostic information when evaluating nonischemic cardiomyopathy. Stress tests either exercise or chemical with or without imaging are commonly used to exclude underlying ischemia. If these studies are inconclusive, the patient may require cardiac catheterization with ventriculography. However, if the patient is low to intermediate likelihood for coronary artery disease, coronary computed tomography angiography can be performed to differentiate between ischemic and nonischemic cardiomyopathy. Positron emission tomography may be utilized for the evaluation of cardiac sarcoidosis.

Cardiac MRI (CMR) is considered the reference standard to assess myocardial anatomy, function, and viability and often reveals the underlying etiology of heart failure [1]. Delayed enhancement in nonischemic cardiomyopathy usually occurs in a mid-myocardial or subepicardial location rather than subendocardial or transmural as seen in ischemic disease. The location of delayed enhancement can also help to guide myocardial biopsy thus increasing yield of a diagnosis. It can also be utilized to screen in genetically positive but phenotypically negative patients to better help with risk stratification. Finally, there has been significant prognostic value shown related to the location and extent of delayed enhancement.

\subsection{Hypertrophic}

Hypertrophic cardiomyopathy is the most common genetic cardiac disorder with heterogeneous phenotypic expression. Patients present clinically most commonly with sudden death from ventricular fibrillation, atrial fibrillation, or heart failure but may be asymptomatic. MRI is utilized not only to make the diagnosis of hypertrophic cardiomyopathy but also to characterize the morphologic types related to the distribution of the hypertrophied muscle. Different types include apical, mid-ventricular, concentric, and mass-like although the most common is asymmetric septal hypertrophy. Ventricular wall thickness of $>30 \mathrm{~mm}$ (normal $<15 \mathrm{~mm}$ ) has 

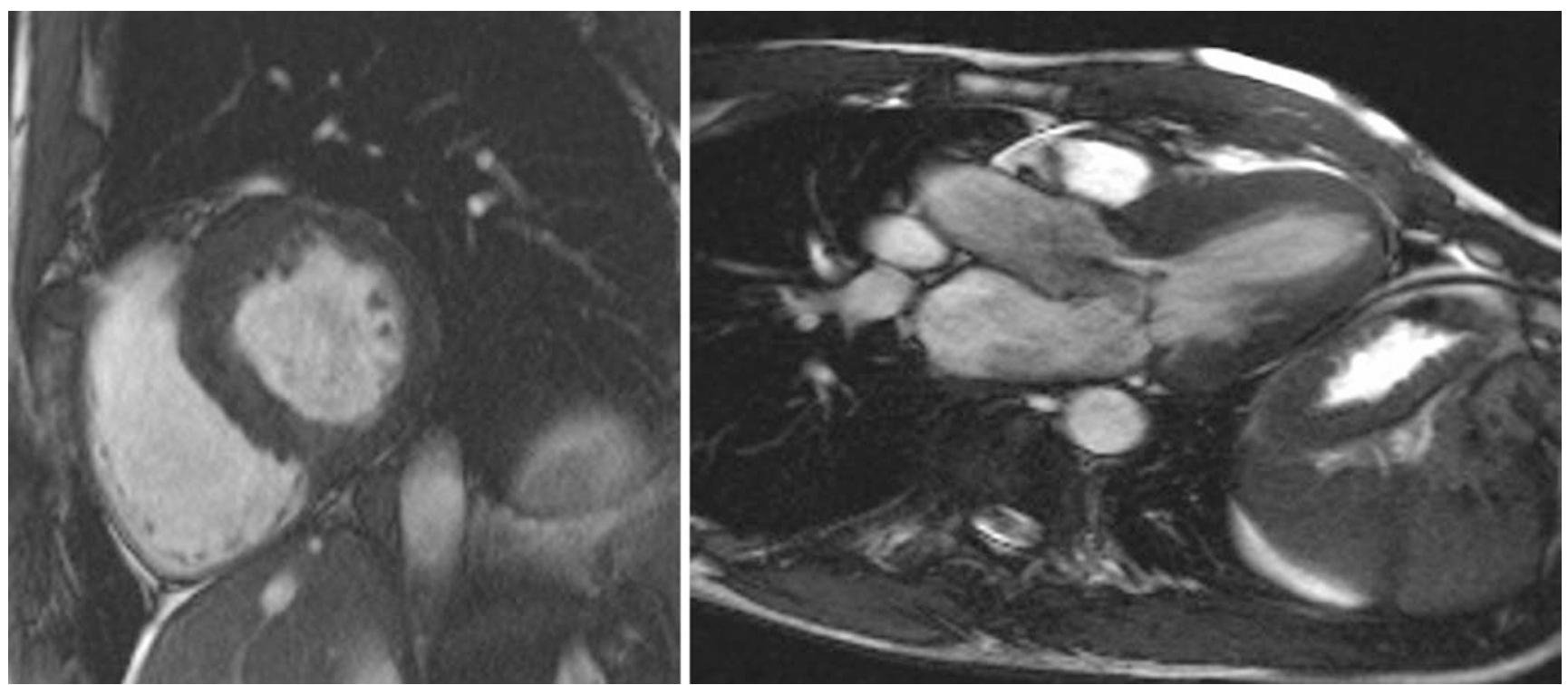

Fig. 16.1 Septal hypertrophic cardiomyopathy. Short axis steady-state free precession image demonstrates thickening of the myocardium involving the anteroseptal segment of the basal left ventricle. Threechamber image from a cine steady-state free precession series demon- strates septal hypertrophy with anterior motion of the anterior leaflet of the mitral valve and associated flow jet in the left ventricular outflow tract below the level of the aortic valve been shown to be an independent risk factor for sudden cardiac death [2]. MRI is the procedure of choice for monitoring left ventricular mass over time and in response to treatment.

The area of hypertrophy in asymmetric septal type is commonly located at the basal aspect of the interventricular septum which can result in left ventricular outflow tract obstruction as shown in Fig. 16.1. Using phase-contrast imaging on MRI, the degree of obstruction can be evaluated based on the velocity in the left ventricular outflow tract using the modified Bernoulli equation (gradient $=4 \mathrm{~V}^{2}$ ) to calculate the gradient (severe $>50 \mathrm{~mm} \mathrm{Hg}$ ). If conservative treatments are not effective, septal ablation or surgical procedures may be required for LVOT obstruction. The accelerated flow in the left ventricular outflow tract can result in systolic anterior motion of the mitral valve which can result in mitral regurgitation. Not only can these two findings be identified during MRI examinations, but the severity of mitral regurgitation can also be quantified. MRI can also be utilized to assess cardiac function and regional wall motion.

Delayed enhancement imaging is performed in all hypertrophic cardiomyopathy patients in order to evaluate for interstitial fibrosis, microfibrillar disarray, or microvascular obstruction. The delayed enhancement images are positive in $60 \%$ of these patients [3]. While the delayed enhancement is most commonly seen in a patchy distribution in the midmyocardium at the right ventricular insertion point, it can be seen throughout the hypertrophied myocardium and sometimes even in the non-hypertrophied muscle. Delayed enhancement has demonstrated prognostic information in several studies of cardiac outcomes including worsening heart failure symptoms, ventricular arrhythmias, ICD discharge, and sudden cardiac death [4]. The absence of delayed enhancement has prognostic information as well with overall lower risk for adverse cardiac outcomes although the risk is still not absent [5]. Not only is the presence or absence of delayed enhancement important, but the extent of late delayed enhancement is a significant predictor of adverse cardiac outcomes [5]. The largest study to date in this patient cohort demonstrated that only the extent of delayed enhancement was a strong predictor of adverse cardiac events and mortality [6]. Limited studies are available regarding the utilization of MRI to monitor treatment for hypertrophic cardiomyopathy.

\section{Key Point}

- In hypertrophic cardiomyopathy, MRI is used not only to make the diagnosis but also to characterize the morphology and evaluate for outflow obstruction.

\subsection{Dilated Cardiomyopathy}

\subsubsection{Idiopathic}

Idiopathic dilated cardiomyopathy represents an end-stage pump failure of the heart; the disease is considered to be idiopathic when no immediate cause is discerned. However, 
it is believed that this end-stage condition of the heart may be produced by a variety of toxic, metabolic, or infectious agents. Idiopathic dilated cardiomyopathy may be the late sequela of infection such as viral myocarditis. In some cases, genetic abnormalities are also associated with this condition. In this condition, there is gradual increased left ventricular end-systolic and end-diastolic volumes with continuously declining ejection fraction. The walls of the ventricle are thinned.

On CMR, decreased function, ventricular dilatation, and wall thinning are readily demonstrated. However, the primary advantage of CMR compared to other imaging modalities is the use of late gadolinium enhancement. Late gadolinium is not seen in all patients. However, in some patients, there is characteristic enhancement if the mid-myocardium in a noncoronary artery distribution. This pattern of late gadolinium enhancement has also been recognized in postmortem hearts as a hallmark of this condition. Right ventricular dilatation is also characteristic of idiopathic dilated cardiomyopathy. Furthermore, the presence and extent of myocardial late enhancement have been shown to predict the clinical outcome [7]. After adjustment for LVEF and other conventional prognostic factors, both the presence of fibrosis and the extent of midwall enhancement on CMR were independently and incrementally associated with all-cause mortality.

\subsubsection{Viral Myocarditis}

The use of CMR for the evaluation of viral myocarditis is increasingly accepted. The diagnosis of viral myocarditis is challenging, as no single test may be used alone for diagnosis. Patient presentation is with chest pain in the setting of a recent or ongoing viral syndrome. The primary differential diagnoses include acute coronary artery syndrome and pericarditis. Other inflammatory myocarditis may also be considered. Troponin values are typically only mildly elevated.

CMR is used for assessment of inflammatory changes such as edema, hyperemia, and myocyte necrosis. No single pathognomonic finding is present. Rather, the Lake Louise criteria are applied. This requires abnormalities in two of the three tissue characterization techniques: T2-weighted images for edema, T1 early gadolinium enhancement imaging for hyperemia, and late gadolinium enhancement for myocyte necrosis and fibrosis. T1 and T2 mapping techniques can help reduce uncertainty that is otherwise associated with qualitative assessment of signal intensity change. A recent multicenter study showed that LV function may be normal or mildly depressed [8]. Overall sensitivity of CMR was $82 \%$. Late gadolinium enhancement may be present in a nonischemic pattern in $80 \%$ of cases. The distribution of LGE is typically either subepicardial or midwall. Pericardial effusions are not common.

\subsubsection{Takotsubo Myocarditis}

Takotsubo is a transient condition involving a distinctive pattern of left ventricular dysfunction. The unusual aspect of the LV dysfunction is its temporal relationship with stressful events, either emotional (e.g., death of a loved one) or physical (e.g., major neurological or abdominal surgery). Patients with this condition recover normal function either days to weeks of the clinical presentation. The underlying etiology is an excess of catecholamines precipitated by a stressful event. The primary differential diagnosis is an acute coronary artery syndrome or possibly viral myocarditis.

The term takotsubo derives from the original Japanese description of the syndrome; there is distinct apical ballooning of the left ventricle that resembles an octopus pot. The ventricular dilation involves multiple coronary territories, beginning in the mid-ventricle but extending into the apex. Ventricular hypokinesis is present, particularly in these dilated myocardial regions. One of the primary CMR features is myocardial edema seen on T2-weighted images. Perfusion images are normal. Typically, LGE images do not show focal lesions that my otherwise be associated with acute myocardial infarction. However, LGE is infrequently reported in less than $10 \%$ of cases [9].

\subsubsection{Alcoholic Cardiomyopathy}

Alcoholic cardiomyopathy is an acquired cardiomyopathy. For diagnosis, documentation of long-term consumption of alcohol at high quantities (>80 g per day over 5 years or more) is required. For many patients, successful clinical history over this period is not available. Like other dilated cardiomyopathies, alcoholic cardiomyopathy is characterized by global ventricular dilatation, decreased myocardial function, and myocardial thinning in its end-stage presentation. Women may be more susceptible than men given equivalent quantities of ingested alcohol. Pathologic changes are similar to idiopathic dilated cardiomyopathy.

No specific CMR findings are present, other than that of abnormal ventricular size and shape. Scar patterns may be assumed to be nonischemic in nature, so that CMR can exclude myocardial scar associated with prior infarction [10]. As opposed to echocardiography, CMR may be useful to exclude other etiologies of cardiomyopathy such ischemic heart disease, iron overload, or amyloidosis.

\subsubsection{Left Ventricular Noncompaction}

Left ventricular noncompaction cardiomyopathy (LVNC) is a rare primary genetic cardiomyopathy characterized by excess myocardial trabeculation more than twice as thick as 
the underlying myocardial wall (Fig. 16.2) [11]. The trabeculae carneae consist of slim columns of myocardial cells at the luminal ventricular surfaces. The function of the trabecula is to increase cardiac output and enable oxygen supply prior to coronary vascularization. During myocardial development, trabeculae compact leading to an increase in thickness of the compact myocardium.

LVNC is associated with heart failure, regional wall motion abnormalities, and myocardial fibrosis. The ratio of the thickness of noncompacted (NC) versus compacted (C) myocardium $(\mathrm{NC} / \mathrm{C})$ is a simple and fast method to quantify trabeculation [12]; a ratio greater than 2.3 to 1 is commonly used as an MRI index of noncompacted myocardium. Although the NC/C ratio is easy to measure, the specificity is low; $33 \%$ of the subjects in the MESA cohort had a NC/C ratio greater than 2.3 [13]. Improved specificity of the $\mathrm{NC} / \mathrm{C}$ ratio is obtained when several segments show high $\mathrm{NC} / \mathrm{C}$ ratios and LV dysfunction is present. In a study by Wan et al., the mean number of compacted segments was 7.4; noncompaction was present in the apical segments in all patients. Forty percent of patients demonstrated a nonischemic pattern of late gadolinium enhancement, most commonly midmyocardial in location [14].

\subsubsection{Collagen Vascular Disease/Connective Tissue Disorders}

Connective tissue disease consists of a heterogeneous group of disorders including systemic lupus erythematosus, systemic sclerosis, and Sjogren's syndrome, as well as overlap

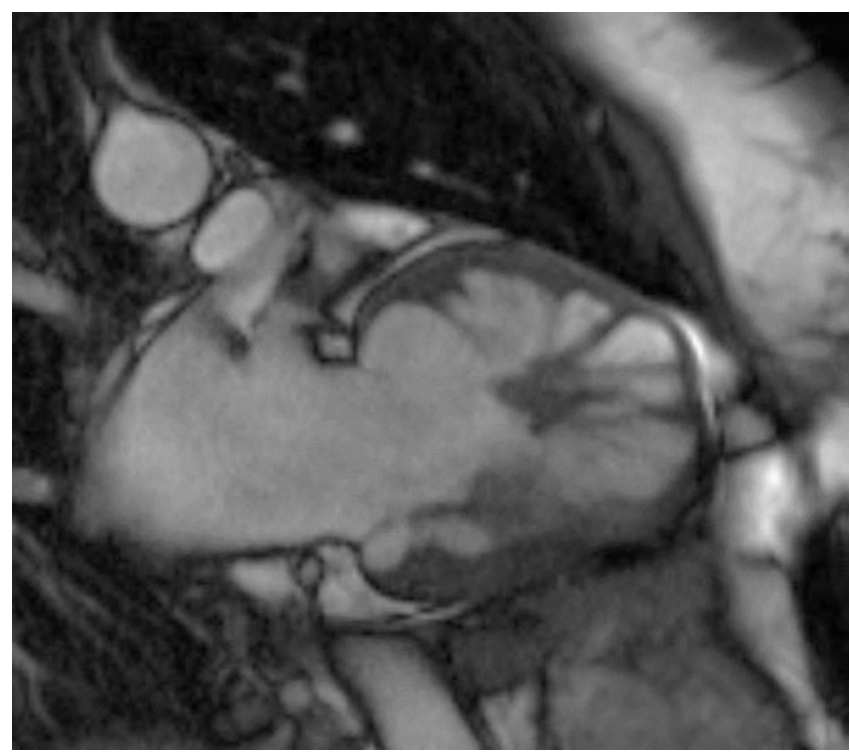

Fig. 16.2 Noncompaction cardiomyopathy. Vertical long axis view of the left ventricle shows a stellate pattern of prominent trabeculation. The left ventricle is dilated, and the myocardium at the apical anterior wall is thinned between these conditions. Cardiac involvement includes abnormalities of valves, myocardium, and pericardium. The underlying common pathologic features are abnormal activation of the immune system, often targeted toward small blood vessels and internal organs.

Myocardial involvement is often subclinical, with diffuse myocardial fibrosis. Ejection fraction is preserved in early stages of disease in the majority of cases. Reports of focal late gadolinium enhancement are highly variable, from 0 (in our own experience) to $60 \%$ of patients [15]; likely this variability depends on the particular subset of patients evaluated. As a result, T1 mapping techniques, including evaluation of extracellular volume fraction, have been evaluated. The primary $\mathrm{T} 1$ mapping findings are (a) increased native $\mathrm{T} 1$, (b) increased extracellular volume fraction, and (c) decreased T1 times after gadolinium administration [16]. However, these findings are not specific, and changes in myocardial T1 mapping parameters are not distinct from other nonischemic cardiomyopathies.

Key Point

- Clinical history, ventricular structure and function, and pattern of late gadolinium enhancement are used to identify subtypes of dilated cardiomyopathy by CMR.

\subsection{Restrictive}

\subsubsection{Amyloid}

Cardiac involvement in amyloidosis is common with deposition of insoluble glycoproteins in the extracellular spaces of myocardial tissue, valve leaflets, and vessels. It can result in restrictive cardiomyopathy which is the leading cause of death in approximately half of amyloid patients.

On cine imaging, there is biventricular myocardial thickening with normal ventricular chamber size, atrial enlargement, and preserved systolic function. Diastolic dysfunction can be demonstrated on mitral inflowing imaging with cine phase-contrast pulse sequence imaging. In the late fibrotic stage, cine images can demonstrate mitral and tricuspid insufficiency.

There is rapid redistribution of gadolinium from the blood pool resulting in altered $\mathrm{T} 1$ kinetics in which the myocardium nulls before the blood pool on inversion time (TI) scout images. The altered kinetics can make selection of an appropriate TI time difficult. Phase-sensitive inversion recovery (PSIR) sequence images can be helpful given that they are independent of the TI value. The prognostic value of delayed enhancement imaging has not shown consistent 


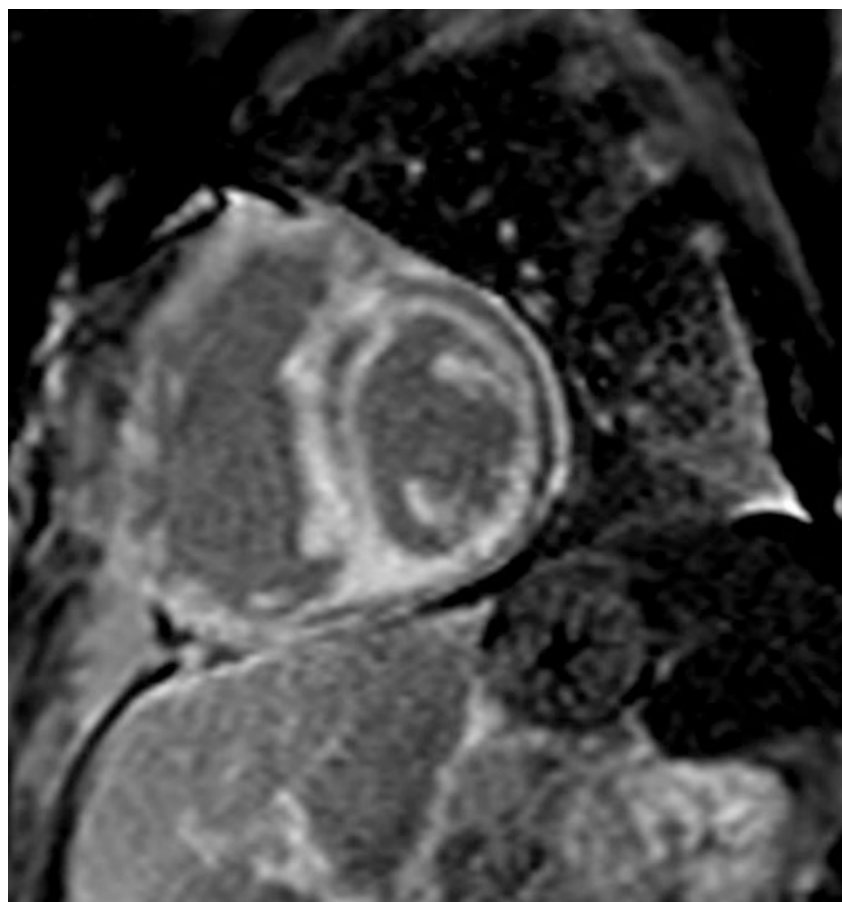

Fig. 16.3 Cardiac amyloid. Short axis delayed enhancement image demonstrates diffuse subendocardial delayed enhancement with more extensive diffuse delayed enhancement in the septum. Note the blood pool is also dark on these images when there is appropriate nulling of the myocardium in the lateral wall which is very characteristic for myocardial amyloidosis

value. The emerging T1 mapping and ECV estimation techniques have shown promise in correlating with cardiac function and risk stratification [17]. The delayed enhancement pattern is quite variable but most often diffuse and irregular in a noncoronary distribution as shown in Fig. 16.3.

CMR has shown promise in differentiating subtypes of cardiac amyloidosis primarily between light chain amyloid and transthyretin-related amyloidosis based on LV mass as well as location and extent of delayed enhancement. The determination of subtype is important given differences in treatment as well as determining prognosis with survival worse for light chain amyloid.

\subsubsection{Sarcoid}

The hallmark of cardiac sarcoidosis is the presence of necrotizing granulomas in the myocardium. In the acute phase, there can be myocardial thickening and edema with delayed enhancement of the mid-myocardium and/or subepicardium. Perfusion or early post contrast T1-weighted images may demonstrate increased contrast enhancement suggesting inflammation with hyperemia. In the chronic phase, edema is absent, but wall thickening and delayed enhancement may be seen. In the burnt-out phase, transmural delayed enhance- ment may be seen [18] with wall thinning. The imaging appearance alone can be difficult to distinguish from myocarditis.

Delayed enhancement has prognostic value as a predictor of death as well as other adverse events such as aborted sudden death, appropriate ICD discharge, and ventricular arrhythmias [19]. It is a stronger predictor than other measures such as LVEF and symptoms. The lack of delayed enhancement also appears to have high negative predicative value with respect to adverse outcomes.

CMR also appears to have value in monitoring disease status during treatment with steroids [20]. Steroid treatment has been associated with decrease in delayed enhancement as well as overall improvement in functional parameters.

The AHA consensus guidelines reserve the recommended use of CMR for diagnosis and differentiation from other cardiomyopathies as well as for functional assessment rather than specifically for risk stratification and prognosis [21].

\section{Key Point}

- Midwall myocardial delayed enhancement is suggestive of an infiltrative process rather than ischemia and raises concern for a restrictive cardiomyopathy.

\subsection{Storage Disease}

\subsubsection{Hemochromatosis}

Systemic iron overload due to hemosiderosis or transfusiondependent anemias can result in dilated or restrictive cardiomyopathy with progressive diastolic and systolic dysfunction. Dysrhythmias are also commonly present.

Myocardial iron deposition, in the form of ferritin and hemosiderin, is demonstrated on gradient-echo images. Utilizing multiple different TE values, the absolute $\mathrm{T} 2 *$ value can be determined which is the best indicator of myocardial iron content [22]. A T2* $<20 \mathrm{~ms}$ is considered diagnostic of significant iron overload. T2* values have been shown to detect iron overload earlier than changes of LVEF and have also been shown to be a strong independent predictor of adverse clinical outcomes such as heart failure, arrhythmias, and sudden cardiac death [22].

CMR is invaluable in monitoring treatment response to chelation therapies demonstrated by improvements in $\mathrm{T} 2 *$ and LVEF [23]. Pennell et al. [24] demonstrated a significantly reduced risk of developing heart failure with improvement in $\mathrm{T} 2 *$ with only minimal improvement in LVEF suggesting LVEF may underestimate the clinical impact of chelation therapies. 
CMR is recognized in the AHA Consensus Statement [25] as a critical tool in the diagnosis and clinical management of iron overload cardiomyopathy.

\subsubsection{Fabry Disease}

Fabry disease is an X-linked recessive disorder of glycosphingolipid metabolism with resultant intracellular accumulation of glycosphingolipids especially in the skin, kidneys, and heart.

There is concentric LV thickening on CMR which can be confused with hypertrophic cardiomyopathy. Mid-myocardial or subepicardial pattern of delayed enhancement may be seen typically involving the basal inferolateral segment. Delayed enhancement has been shown to be associated with development of ventricular arrhythmias as well as sudden cardiac death [26]. Rather than the presence or absence of delayed enhancement, it was the annual increase in fibrosis demonstrated on delayed enhancement images that was an independent predictor of ventricular arrhythmias.

T1 mapping technique has been utilized to characterize Fabry's cardiomyopathy. Reduction in T1 values are associated with reduced longitudinal strain and early diastolic dysfunction prior to the development of LV hypertrophy. This suggests T1 mapping may be valuable in detecting early dysfunction before structural abnormalities [27]. CMR has also been utilized to monitor response to enzyme replacement therapies. One study demonstrated that despite enzyme replacement therapy, there can be progression of delayed enhancement suggesting progressive fibrosis [26].

\section{Key Point}

- CMR is a critical tool in the diagnosis and in monitoring treatment of iron overload cardiomyopathy.

\subsection{Arrhythmogenic Right Ventricular Cardiomyopathy/Dysplasia (ARVC/D)}

Arrhythmogenic right ventricular cardiomyopathy (ARVC) is a heritable heart muscle disease characterized by fibrofatty replacement of predominantly the right ventricular (RV) myocardium. This predisposes patients to potentially lifethreatening arrhythmias and ventricular dysfunction. Affected patients typically present between the second and fourth decade of life with arrhythmias coming from the RV2. ARVC is an unusual condition, with an estimated prevalence of 1 in 1000 to 1 in 5000 Caucasian individuals. Approximately $60 \%$ of index cases harbor mutations in genes encoding the cardiac desmosome, a structure that provides mechanical connection between cardiomyocytes. The defective mechanical connections in ARVC may structurally be represented by global or regional contraction abnormalities, ventricular enlargement, and/or fibrofatty replacement. As such, accurate evaluation of cardiac morphology and function is essential for ARVC diagnosis and management.

ARVC is generally transmitted as an autosomal dominant trait with incomplete penetrance and variable expressivity. However, it is important to realize that $50-70 \%$ of mutation carriers will never develop disease expression and that severity of disease may vary greatly, even among members of the same family or those carrying the same mutation. In contrast, a negative genetic test result in a proband does not exclude the possibility of disease, nor does it exclude the possibility of a genetic process in the individual or family.

The CMR protocol for ARVC is somewhat unique compared to other cardiomyopathies. The primary difference is the addition of axial black blood T1-weighted fast spin echo images. These cover the left and right ventricles from the base to the diaphragm. Cine SSFP axial images are then obtained in the same plane, with the same slice thickness and gap ( $8 \mathrm{~mm} \times 2 \mathrm{~mm}$ is typically used). After this, standard SSFP cine imaging of the ventricles is obtained in the short and long axes. Many sites also obtain a vertical long axis cine view of the right ventricle. After gadolinium administration, short and long axis late gadolinium enhancement images are obtained. In patients with significant ventricular ectopy, a low dose of a beta-blocker (metoprolol 25-50 mg) is recommended for arrhythmia suppression during the CMR scan.

The diagnostic TFC for CMR require the presence of both qualitative findings (RV regional akinesia, dyskinesia, dyssynchronous contraction) and quantitative metrics (decreased ejection fraction or increased indexed RV end-diastolic volume) (Table 16.1).

Besides global reduction in RV function, more subtle regional disease of the RV has been described in the literature using a variety of terms including focal bulges, microaneurysms, segmental dilatation, regional hypokinesis, etc. In

Table 16.1 MRI task force criteria for ARVC

Global or regional dysfunction and structural alterations
Region
contraction AND one of the following:
- $\mathrm{RV}$ EDV/BSA $\geq 110 \mathrm{~mL} / \mathrm{m}^{2}$ (male) or $\geq 100 \mathrm{~mL} / \mathrm{m}^{2}$ (female)
- $\mathrm{RV}$ ejection fraction $\leq 40 \%$
Minor
Regional RV akinesia or dyskinesia or dyssynchronous $\mathrm{RV}$
contraction AND one of the following:
- $\quad \mathrm{RV}$ EDV/BSA $\geq 100$ to $110 \mathrm{~mL} / \mathrm{m}^{2}$ (male) or $\geq 90$ to $100 \mathrm{~mL} / \mathrm{m}^{2}$
(female)
$-\quad \mathrm{RV}$ ejection fraction $>40$ to $\leq 45 \%$
$E D V$ end-diastolic volume, $B S A$ body surface area, $R V$ right ventricle


the current TFC, the terms "akinesia" (lack of motion) and "dyskinesia" (abnormal movement, instead of contracting in systole, the myocardium bulges outward in systole) and "dyssynchronous" (regional peak contraction occurring at different times in adjacent myocardium) are used for all imaging modalities (CMR, echocardiography and angiography) to describe regional wall motion abnormalities in ARVC. The RV apex is spared in early ARVC. The so-called accordion sign represents a focal "crinkling" of the myocardium in the subtricuspid region [28] (Fig. 16.4). In terms of TFC, the accordion sign is due to a small region of highly localized myocardium with dyssynchronous contraction.

RV LGE has been observed in up to $88 \%$ of ARVC patients, while LV LGE was reported in up to $61 \%$ of cases. In ARVC, RV wall thinning is pronounced, which makes the LGE technique less reliable than for the LV. Second, distinguishing fat from fibrosis by LGE sequences is challenging, which makes its interpretation highly subject to the CMR physician's experience.

Some ARVC subjects have early and predominant LV involvement [29]. LV involvement has even been reported in $76 \%$ of ARVC subjects, of whom the majority had advanced disease. The disease is, therefore, increasingly being referred to as "arrhythmogenic cardiomyopathy." LV involvement in ARVC typically manifests as LGE, often involving the inferior and lateral walls without concomitant wall motion abnormalities. Figure 16.5 shows an example. Septal LGE is

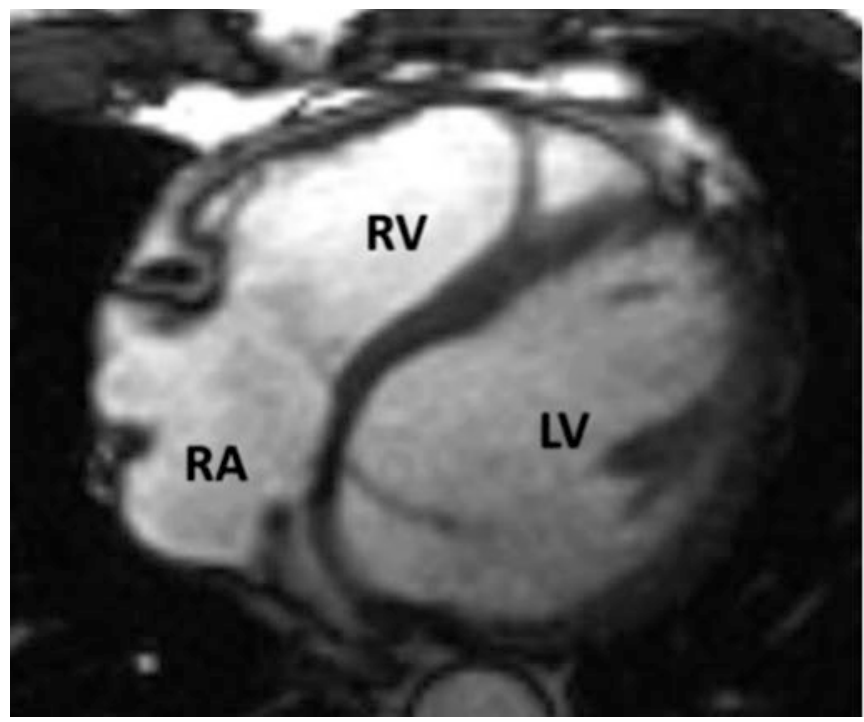

Fig. 16.4 Arrhythmogenic right ventricular cardiomyopathy. Enddiastolic (left) and end-systolic (right) images are shown. In systole at the arrows, there is infolding of the right ventricle wall, termed the present in more than $50 \%$ of cases with left dominant ARVC, in contrast to the right dominant pattern in which septal involvement is unusual. In addition, LV fatty infiltration was shown to be a prevalent finding in ARVC, often involving the subepicardial lateral LV and resulting in myocardial wall thinning [30].

\section{Key Point}

- ARVC typically manifests as right heart dysfunction and dilation out of proportion to similar findings in the left heat.

\subsection{Concluding Remarks}

Nonischemic cardiomyopathy encompasses a heterogeneous group of diseases. Genetic components of these disease are increasingly being recognized. The myocardium responds to injury by accumulation of either fibrotic tissue or fatty replacement or both. MRI is very good at detecting fatty tissue; the detection of myocardial fibrosis is performed using late gadolinium enhancement techniques and/or T1 mapping. While all patients undergo echocardiography screening to assess for myocardial function, tissue characterization remains in the realm of cardiac MRI evaluation.

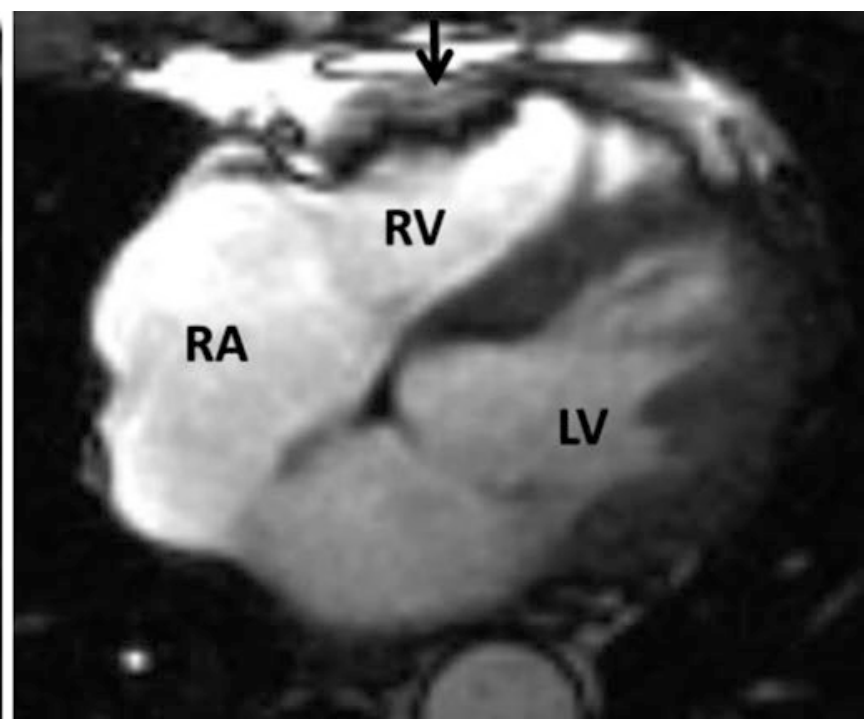

accordion sign. This is due to dyskinesia of the right ventricle free wall in the subtricuspid region 


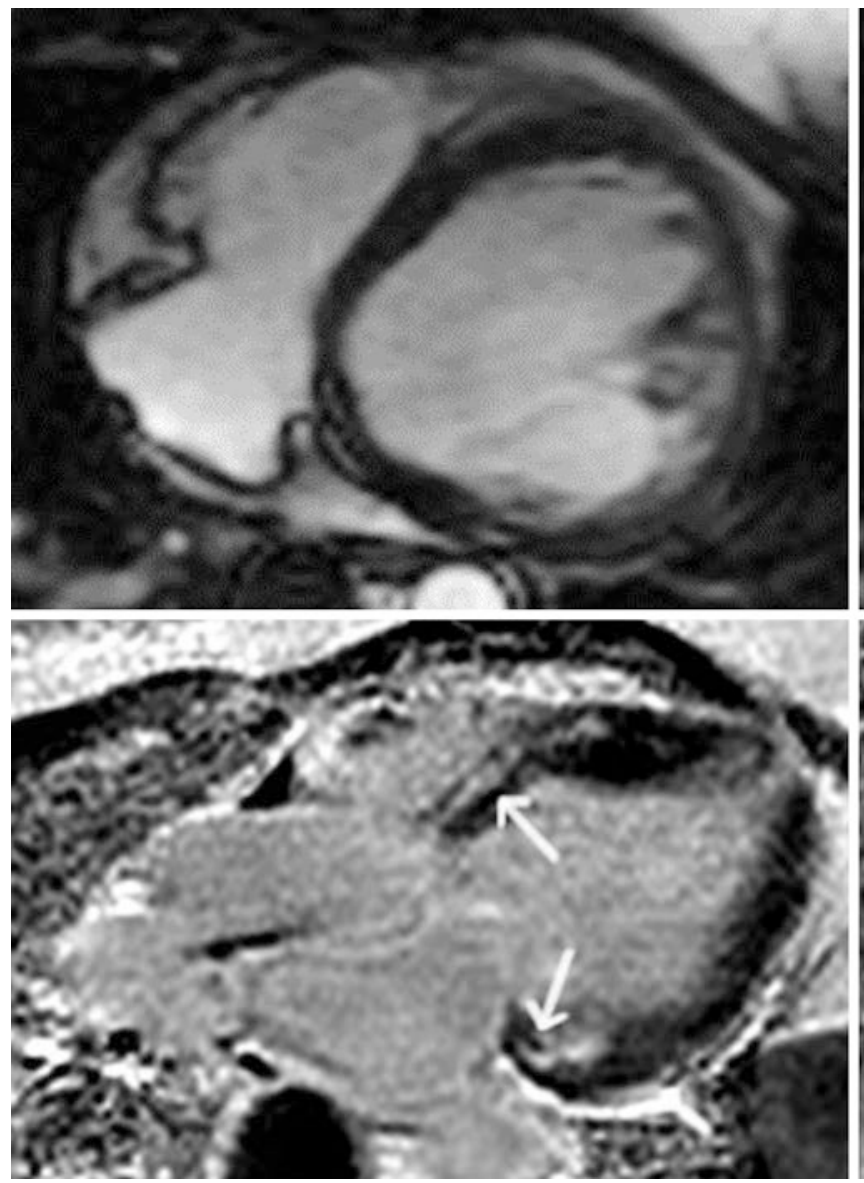

Fig. 16.5 Left ventricular presentation of arrhythmogenic right ventricular cardiomyopathy. Top row shows images at end diastole (left) and end systole (right), with poor left ventricular ejection fraction and left ventricular enlargement. Bottom row shows late gadolinium

\section{Take-Home Messages}

- Late gadolinium enhancement shows nonischemic pattern of enhancement in cardiomyopathies, characterized by midwall or epicardial enhancement in a noncoronary artery distribution.

- T1 mapping may be used to assess myocardial fibrosis. Typically, native T1 values are elevated due to the presence of fibrosis, while post gadolinium T1 values are lower than expected.

- In addition to late gadolinium enhancement images, myocardial global and regional function is readily assessed to further characterize the myocardial abnormality and extent in nonischemic cardiomyopathy.

\section{Bibliography}

1. Karamitsos TD, Francis JM, Myerson S, Selvanayagam JB, Neubauer S. The role of cardiovascular magnetic resonance imaging in heart failure. J Am Coll Cardiol. 2009;54(15):1407-24. https://doi.org/10.1016/j.jacc.2009.04.094.

2. Spirito P, Bellone P, Harris KM, Bernabo P, Bruzzi P, Maron BJ. Magnitude of left ventricular hypertrophy and risk
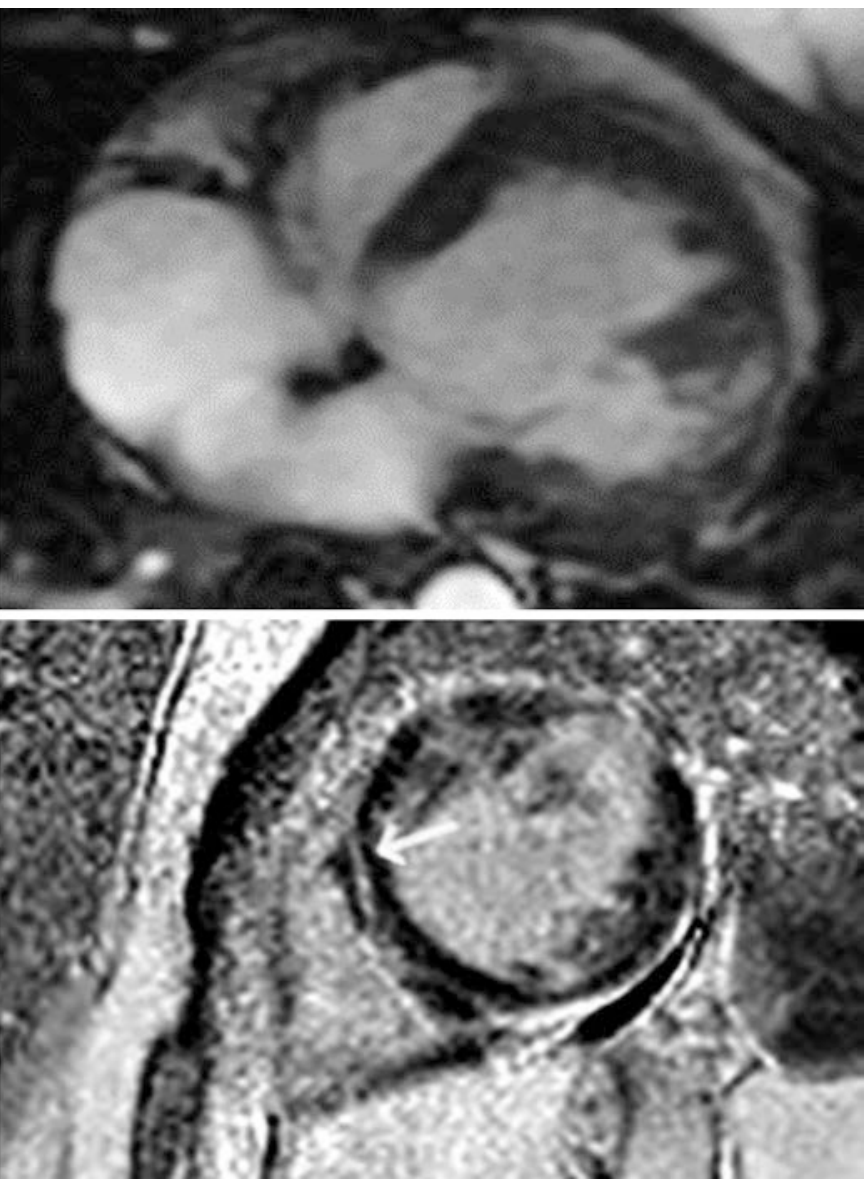

enhancement images, with focal enhancement on the four-chamber view (left) at the base (arrows) of the left ventricle. The short axis view (right) shows scattered late gadolinium enhancement (arrow)

of sudden death in hypertrophic cardiomyopathy. $\mathrm{N}$ Engl J Med. 2000;342(24):1778-85. https://doi.org/10.1056/ NEJM200006153422403.

3. Green JJ, Berger JS, Kramer CM, Salerno M. Prognostic value of late gadolinium enhancement in clinical outcomes for hypertrophic cardiomyopathy. J Am Coll Cardiol Img. 2012;5(4):370-7. https:// doi.org/10.1016/j.jcmg.2011.11.021.

4. Rubinshtein R, Glockner JF, Ommen SR, Araoz PA, Ackerman MJ, Sorajja P, et al. Characteristics and clinical significance of late gadolinium enhancement by contrast-enhanced magnetic resonance imaging in patients with hypertrophic cardiomyopathy. Circ Heart Fail. 2010;3(1):51-8. https://doi.org/10.1161/ CIRCHEARTFAILURE.109.854026.

5. Chan RH, Maron BJ, Olivotto I, Pencina MJ, Assenza GE, Haas $\mathrm{T}$, et al. Prognostic value of quantitative contrast-enhanced cardiovascular magnetic resonance for the evaluation of sudden death risk in patients with hypertrophic cardiomyopathy. Circulation. 2014;130(6):484-95. https://doi.org/10.1161/ CIRCULATIONAHA.113.007094.

6. Ismail TF, Jabbour A, Gulati A, Mallorie A, Raza S, Cowling TE, et al. Role of late gadolinium enhancement cardiovascular magnetic resonance in the risk stratification of hypertrophic cardiomyopathy. Heart. 2014;100(23):1851-8. https://doi.org/10.1136/ heartjnl-2013-305471.

7. Gulati A, Jabbour A, Ismail TF, Guha K, Khwaja J, Raza S, et al. Association of fibrosis with mortality and sudden cardiac death in patients with nonischemic dilated cardiomyopathy. JAMA. 2013;309(9):896-908. https://doi.org/10.1001/ jama.2013.1363. 
8. Banka P, Robinson JD, Uppu SC, Harris MA, Hasbani K, Lai WW, et al. Cardiovascular magnetic resonance techniques and findings in children with myocarditis: a multicenter retrospective study. J Cardiovasc Magn Reson. 2015;17:96. https://doi.org/10.1186/ s12968-015-0201-6.

9. Placido R, Cunha Lopes B, Almeida AG, Rochitte CE. The role of cardiovascular magnetic resonance in takotsubo syndrome. J Cardiovasc Magn Reson. 2016;18(1):68. https://doi.org/10.1186/ s12968-016-0279-5.

10. Francone M. Role of cardiac magnetic resonance in the evaluation of dilated cardiomyopathy: diagnostic contribution and prognostic significance. ISRN Radiol. 2014;2014:365404. https://doi. org/10.1155/2014/365404.

11. Maron BJ, Towbin JA, Thiene G, Antzelevitch C, Corrado D, Arnett D, et al. Contemporary definitions and classification of the cardiomyopathies: an American Heart Association Scientific Statement from the Council on Clinical Cardiology, Heart Failure and Transplantation Committee; Quality of Care and Outcomes Research and Functional Genomics and Translational Biology Interdisciplinary Working Groups; and Council on Epidemiology and Prevention. Circulation. 2006;113(14):180716. https://doi.org/10.1161/CIRCULATIONAHA.106.174287. CIRCULATIONAHA.106.174287 [pii].

12. Petersen SE, Selvanayagam JB, Wiesmann F, Robson MD, Francis $\mathrm{JM}$, Anderson RH, et al. Left ventricular non-compaction. J Am Coll Cardiol. 2005;46(1):101-5. https://doi.org/10.1016/j. jacc.2005.03.045.

13. Kawel N, Nacif M, Arai AE, Gomes AS, Hundley WG, Johnson WC, et al. Trabeculated (noncompacted) and compact myocardium in adults: the multi-ethnic study of atherosclerosis. Circ Cardiovasc Imaging. 2012;5(3):357-66. https://doi.org/10.1161/ CIRCIMAGING.111.971713.

14. Wan J, Zhao S, Cheng H, Lu M, Jiang S, Yin G, et al. Varied distributions of late gadolinium enhancement found among patients meeting cardiovascular magnetic resonance criteria for isolated left ventricular non-compaction. J Cardiovasc Magn Reson. 2013;15:20. https://doi.org/10.1186/1532-429X-15-20.

15. Puntmann VO, D'Cruz D, Smith Z, Pastor A, Choong P, Voigt $\mathrm{T}$, et al. Native myocardial $\mathrm{T} 1$ mapping by cardiovascular magnetic resonance imaging in subclinical cardiomyopathy in patients with systemic lupus erythematosus. Circ Cardiovasc Imaging. 2013;6(2):295-301. https://doi.org/10.1161/ CIRCIMAGING.112.000151.

16. Mayr A, Kitterer D, Latus J, Steubing H, Henes J, Vecchio F, et al. Evaluation of myocardial involvement in patients with connective tissue disorders: a multi-parametric cardiovascular magnetic resonance study. J Cardiovasc Magn Reson. 2016;18(1):67. https://doi. org/10.1186/s12968-016-0288-4.

17. Banypersad SM, Fontana M, Maestrini V, Sado DM, Captur G, Petrie A, et al. T1 mapping and survival in systemic lightchain amyloidosis. Eur Heart J. 2015;36(4):244-51. https://doi. org/10.1093/eurheartj/ehu444.

18. Vignaux O. Cardiac sarcoidosis: spectrum of MRI features. AJR Am J Roentgenol. 2005;184(1):249-54. https://doi.org/10.2214/ ajr.184.1.01840249.

19. Greulich S, Deluigi CC, Gloekler S, Wahl A, Zurn C, Kramer U, et al. CMR imaging predicts death and other adverse events in suspected cardiac sarcoidosis. J Am Coll Cardiol Img. 2013;6(4):50111. https://doi.org/10.1016/j.jcmg.2012.10.021.
20. Vignaux O, Dhote R, Duboc D, Blanche P, Dusser D, Weber S, et al. Clinical significance of myocardial magnetic resonance abnormalities in patients with sarcoidosis: a 1-year follow-up study. Chest. 2002;122(6):1895-901.

21. Hendel RC, Patel MR, Kramer CM, Poon M, Hendel RC, Carr JC, et al. ACCF/ACR/SCCT/SCMR/ASNC/NASCI/SCAI/SIR 2006 appropriateness criteria for cardiac computed tomography and cardiac magnetic resonance imaging: a report of the American College of Cardiology Foundation Quality Strategic Directions Committee Appropriateness Criteria Working Group, American College of Radiology, Society of Cardiovascular Computed Tomography, Society for Cardiovascular Magnetic Resonance, American Society of Nuclear Cardiology, North American Society for Cardiac Imaging, Society for Cardiovascular Angiography and Interventions, and Society of Interventional Radiology. J Am Coll Cardiol. 2006;48(7):1475-97. https://doi.org/10.1016/j. jacc.2006.07.003.

22. Anderson LJ, Holden S, Davis B, Prescott E, Charrier CC, Bunce $\mathrm{NH}$, et al. Cardiovascular T2-star (T2*) magnetic resonance for the early diagnosis of myocardial iron overload. Eur Heart J. 2001;22(23):2171-9.

23. Ambati SR, Randolph RE, Mennitt K, Kleinert DA, Weinsaft JW, Giardina PJ. Longitudinal monitoring of cardiac siderosis using cardiovascular magnetic resonance $\mathrm{T} 2 *$ in patients with thalassemia major on various chelation regimens: a 6-year study. Am J Hematol. 2013;88(8):652-6. https://doi.org/10.1002/ajh.23469.

24. Pennell DJ, Carpenter JP, Roughton M, Cabantchik Z. On improvement in ejection fraction with iron chelation in thalassemia major and the risk of future heart failure. J Cardiovasc Magn Reson. 2011;13:45. https://doi.org/10.1186/1532-429X-13-45.

25. Pennell DJ, Udelson JE, Arai AE, Bozkurt B, Cohen AR, Galanello $\mathrm{R}$, et al. Cardiovascular function and treatment in beta-thalassemia major: a consensus statement from the American Heart Association. Circulation. 2013;128(3):281-308. https://doi.org/10.1161/ CIR.0b013e31829b2be6.

26. Kramer J, Niemann M, Stork S, Frantz S, Beer M, Ertl G, et al. Relation of burden of myocardial fibrosis to malignant ventricular arrhythmias and outcomes in Fabry disease. Am J Cardiol. 2014;114(6):895-900. https://doi.org/10.1016/j. amjcard.2014.06.019.

27. Pica S, Sado DM, Maestrini V, Fontana M, White SK, Treibel $\mathrm{T}$, et al. Reproducibility of native myocardial $\mathrm{T} 1$ mapping in the assessment of Fabry disease and its role in early detection of cardiac involvement by cardiovascular magnetic resonance. J Cardiovasc Magn Reson. 2014;16:99. https://doi.org/10.1186/ s12968-014-0099-4.

28. Dalal D, Tandri H, Judge DP, Amat N, Macedo R, Jain R, et al. Morphologic variants of familial arrhythmogenic right ventricular dysplasia/cardiomyopathy a genetics-magnetic resonance imaging correlation study. J Am Coll Cardiol. 2009;53(15):1289-99. https:// doi.org/10.1016/j.jacc.2008.12.045.

29. Sen-Chowdhry S, Syrris P, Prasad SK, Hughes SE, Merrifield $\mathrm{R}$, Ward $\mathrm{D}$, et al. Left-dominant arrhythmogenic cardiomyopathy: an under-recognized clinical entity. J Am Coll Cardiol. 2008;52(25):2175-87. https://doi.org/10.1016/j.jacc.2008.09.019.

30. Rastegar N, Zimmerman SL, Te Riele AS, James C, Burt JR, Bhonsale A, et al. Spectrum of biventricular involvement on CMR among carriers of ARVD/C-associated mutations. J Am Coll Cardiol Img. 2015;8(7):863-4. https://doi.org/10.1016/j.jcmg.2014.09.009.

Open Access This chapter is licensed under the terms of the Creative Commons Attribution 4.0 International License (http://creativecommons. org/licenses/by/4.0/), which permits use, sharing, adaptation, distribution and reproduction in any medium or format, as long as you give appropriate credit to the original author(s) and the source, provide a link to the Creative Commons license and indicate if changes were made.

The images or other third party material in this chapter are included in the chapter's Creative Commons license, unless indicated otherwise in a credit line to the material. If material is not included in the chapter's Creative Commons license and your intended use is not permitted by statutory regulation or exceeds the permitted use, you will need to obtain permission directly from the copyright holder. 